\title{
ROLE DE LA PROLACTINE DANS LES MODIFICATIONS DU PARASITISME INDUITES PAR LA LACTATION
}

\author{
J. IBAILENGER*, P. MARCEL*
}

\begin{abstract}
RÉSUME. Afln d'établir le rôle joué par la prolactine dans le retentissement de la lactation sur l'évolution de Slrongyloides ratti chez la ratte, les auteurs essaient de reproduire les effets de cet état physiologique par un traitement avec le sulpiride (benzamide substitué) dont l'effet prolactinogène est intense chez la ratte. Ils notent que les doses faibles reproduisent partiellement les effets de lactation sur le parasitisme en diminuant son intensité et en retardant le début du déparasitage sans toutefois empêcher celui-ci. L'action pharmacologique du sulpiride, qui agit en bloquant les récepteurs à la dopamine, permet d'expliquer que seules les doses faibles agissent et qu'elles limitent leur action au début du parasitisme. Cette expérimentation apporte des arguments en faveur de l'hypothèse attribuant à la prolactine un rôle fondamental dans les modifications de l'évolution de Strongyloides ratti pendant la lactation.
\end{abstract}

Mols-clés : Strongyloides ralti. Prolactine, Lactation, Déparasitage spontané.

\section{The role of prolactin in modifications of parasitism induced by lactation.}

SUMMARY. In order to establish the role played by prolactin in lactation during the evolution of Strongyloides ralli in the female rat, the authors tried to reproduce the effects of this physiological state by treatment with sulpiride (substituted benzamide), of which the prolactinogenic effect is intense in the female rat. Low closes partially reproduced the effects of lactation on parasitism by diminishing its intensity and delaying the onset of rejection, although without preventing it. The pharmacological action of sulpiride, which acts by blocking the dopamine receptors, explains why only low doses are active and why their action is limited to the onset of parasitism. This experiment lends weight to the hypothesis that prolactin plays a fundamental role in modifications of the evolution of Strongyloides ralti during lactation.

Key-words: Slrongyloides ralli. Prolactin. Lactation. Spontaneous cure.

\section{Introduction}

De nombreux travaux mettent en évidence des modifications du parasitisme en fin de grossesse et pendant la lactation. Elles consistent, notamment, pour Plasmodium en une intensification de la parasitémie (Bray et Anderson, 1979;

* U. E. R. Pharmacie, place de la Victoire, F 33076 Bordeaux Cedex.

Accepté le 2 février 1987. 
Van Zon et Eling, 1980) et, pour de nombreuses espèces d'helminthes, elles se traduisent par une augmentation de la ponte, décrite sous le nom de "parturientrise " (Crofton, 1958), ainsi que par un ralentissement du déparasitage spontané.

Le mécanisme par lequel la grossesse et la lactation affectent les infections parasitaires n'est pas bien compris. Il est vraisemblable que plusieurs facteurs interfèrent et que leur intervention diffère selon qu'il s'agisse de la grossesse ou de la lactation. Nous nous intéresserons plus particulièrement à la lactation.

Différents travaux, essentiellement effectués sur le parasitisme du Rat par Nippostrongylus brasiliensis, ont analysé les mécanismes immunitaires susceptibles d'être incriminés. Ils montrent que les femelles lactantes développent des anticorps protecteurs à des titres comparables à ceux trouvés chez les femelles non lactantes (Connan, 1973 ; Connan, 1976 ; Selby et Wakelin, 1975), élaborent des cellules lymphoïdes potentiellement immunes (Dineen et Kelly, 1972), présentent une réponse mastocytaire normale (Connan, 1973; O'Sullivan, 1974) et manifestent une résistance marquée à une réinfection (O'Sullivan et Donald, 1970; Connan, 1972). Néanmoins, pour la plupart des auteurs, le retentissement de la lactation sur le déparasitage s'expliquerait par une immuno-dépression, sans qu'existe un accord général sur les types de réponses immunitaires inhibées, d'autant que les résultats sont parfois contradictoires d'une espèce à l'autre. Cependant, de nombreuses expériences montrent une dépression de la réponse immunitaire des cellules $\mathrm{T}$, associée à la présence de cellules suppressives $\mathrm{T}$ et des facteurs hydrosolubles qu'elles libèrent (Birkeland et Kristoffersen, 1980 ; Bullmer et Hancock, 1977 ; Burrells et coll., 1978 ; Chaouat et Voisin, 1979 ; Chaouat et Voisin, 1981 ; Chaouat et coll., 1979 ; Ruitenberg et Buys, 1980 ; Stahn et coll., 1977 ; Suzuki et Tomasi, 1979).

Parallèlement à ces processus immunitaires, la grossesse et la lactation impliquent un équilibre hormonal particulier : l'œstradiol, aux concentrations présentes dans le sérum des femelles gestantes ne semble pas influencer l'immunité, contrairement à la progestérone et à la prolactine qui auraient un rôle immunodépressif important (Clemens et coll, 1979; Szekeres et coll., 1981; Truycotte et coll., 1968). Aussi, de nombreux auteurs attribuent à la prolactine une fonction primordiale dans l'inhibition du déparasitage spontané provoquée par la lactation.

Ce travail veut essayer d'apporter une contribution à la connaissance du rôle que la prolactine jouerait dans les modifications du parasitisme notées au cours de la lactation. Nous choisissons l'infection du Rat à Strongyloides ratti, sur laquelle nous avons précédemment étudié les répercussions de la lactation (Bailenger et Cabannes, 1976) et nous provoquons une sécrétion de prolactine par un traitement avec le sulpiride* dont l'activité prolactinogène chez le Rat est intense. Elle se traduit dans les deux sexes par une hypertrophie des glandes mammaires avec lactogenèse (Mancini, 1976 ; Stefan et Benakis, 1976) ; chez la ratte, le sulpiride perturbe le cycle œstral avec présence de nombreux et volumineux corps jaunes

\footnotetext{
* Médicament neuroleptique du groupe des benzamides substituées.
} 
fonctionnels comparables à ceux qui caractérisent une lactation physiologique (Lanza et coll., 1971 ; Marchandise, 1971 ; Clemens et coll., 1974 ; Mori et coll., 1975 ; Iwasaki et coll., 1976 ; Soulairac et coll., 1975).

Les répercussions du traitement sont suivies parallèlement sur le parasitisme et sur le taux plasmatique de la corticostérone, en raison de l'importance de cette hormone dans les réactions de défense de l'organisme.

\section{Matériel et méthodes}

Les rats sont de souche Wistar, de sexe femelle, adultes $(180 \pm 20 \mathrm{~g})$ et vierges. Les infestations par $S$. $r$., la surveillance du parasitisme et le dosage de la corticostéronémie ont été précédemment décrits (Bailenger et Guy, 1982).

Les animaux, soumis à une modalité expérimentale déterminée, sont groupés en un lot et les résultats correspondent à une valeur moyenne pour le lot considéré : les œufs et les larves sont numérés dans des échantillons fécaux constitués, à parties égales, par les selles des différents rats d'un même lot ; de même, la corticostéronémie est dosée sur un échantillon de plasma constitué par des volumes égaux du plasma de tous les animaux d'un même lot.

Traitement : le sulpiride est injecté par voie sous-cutanée; la dose journalière est fractionnée en 3 fois en raison de la vie plasmatique du sulpiride qui est de 6 à 8 heures environ. Le traitement est commencé 3 à 5 jours avant l'infestation, selon les expériences, et il est poursuivi, sans interruption -7 jours sur 7 pendant toute la durée du parasitisme.

Les rats témoins reçoivent dans les mêmes conditions, le même volume d'eau distillée stérile, qui sert de solvant au médicament.

\section{Résultats}

\section{Parasitisme}

Expérience 1 (lableau $I$, fig. 1). Une dose quotidienne de $50 \mathrm{mg} / \mathrm{kg}$ ne retentit pas sur l'évolution du parasitisme. $25 \mathrm{mg} / \mathrm{kg}$ diminuent l'intensité du parasitisme et retardent le début du déparasitage dont le terme n'est pas modifié. $10 \mathrm{mg} / \mathrm{kg}$ accentuent les modifications précédentes avec un nombre maximal d'œufs par gramme de selles de $56.10^{3}$ au lieu de $124.10^{3}$ chez les rattes non traitées et un début de déparasitage aux environs du $11^{\mathrm{e}}$ jour au lieu du $8^{\mathrm{e}}$; le déparasitage se termine en même temps que celui des animaux non traités.

Expérience 2 (fig. 1). Une nouvelle expérience avec un traitement à $10 \mathrm{mg} / \mathrm{kg}$ confirme la réduction du parasitisme et nous permet de préciser le début du déparasitage au $10^{\mathrm{e}}$ jour au lieu du $8^{\mathrm{e}}$. 


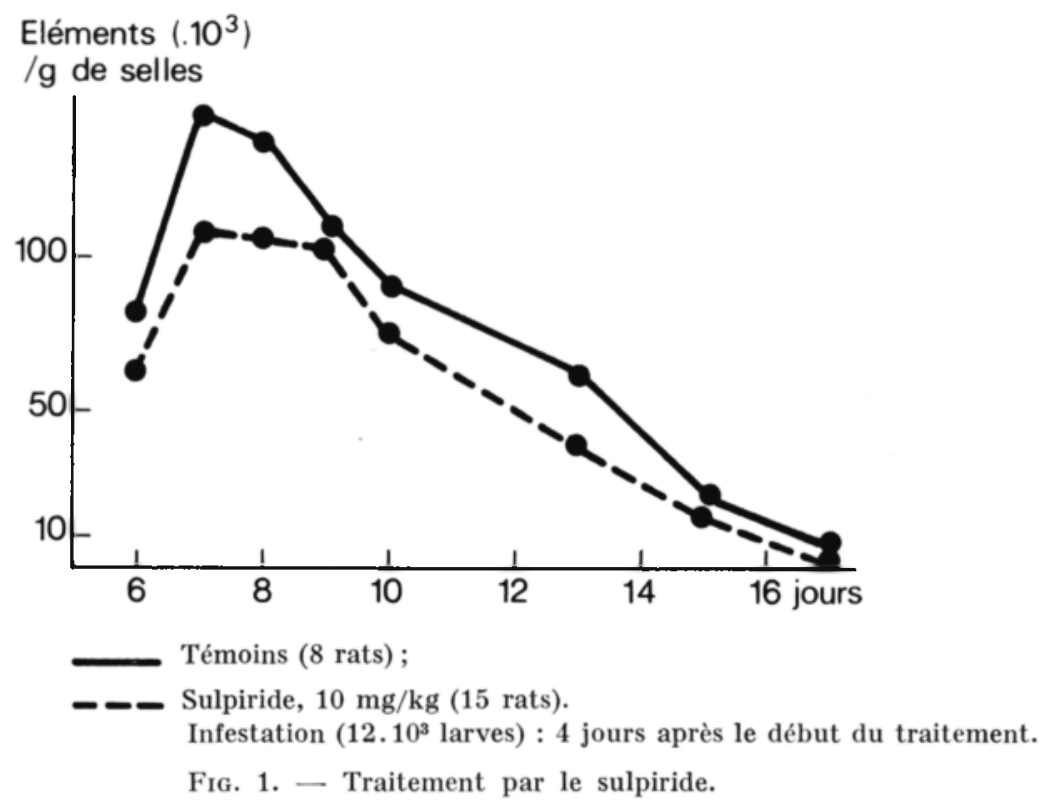

Tableau I. - Traitement par le sulpiride (Exp. 1).

Nombre d'œufs et de larves $\left(.10^{3}\right)$ par gramme de selles.

\begin{tabular}{ccccc}
\hline Temps (jours) & \multicolumn{4}{c}{ Sulpiride $(\mathrm{mg} / \mathrm{kg})$} \\
\cline { 2 - 5 } & 0 & 50 & 25 & 10 \\
\hline 5 & 16 & 12 & 16 & 11 \\
6 & 82 & 95 & 67 & 56 \\
7 & 124 & 111 & 77 & 52 \\
8 & 100 & 100 & 78 & 49 \\
11 & 41 & 53 & 50 & 43 \\
13 & 36 & 26 & 25 & 24 \\
14 & 32 & 22 & 16 & 15 \\
15 & 21 & 10 & 11 & 10 \\
18 & 2 & 2 & 0,4 & 1 \\
20 & 0,6 & 0 & 0,6 & 0,6 \\
Nb rats & 5 & 5 & 5 & 5 \\
\hline
\end{tabular}

Infestation : $12.10^{3}$ larves.

Début du traitement : 3 jours avant infestation.

\section{Corticosléronémie (lableau II)}

La seule modification notable provoquée par les injections quotidiennes et fractionnées de $10 \mathrm{mg} / \mathrm{kg}$ de sulpiride porte sur l'hypercorticostéronémie qui se produit au début de l'infestation. Le traitement ne l'empêche pas mais l'atténue : il diminue son intensité et en limite la durée. 
Tableau II. - Corticostéronémie $(\mu \mathrm{g} / 100 \mathrm{ml})$

des rattes non traitées $(0)$ ou traitées $p a r 10 \mathrm{mg} / \mathrm{kg} / \mathrm{j}$ de sulpiride (S).

\begin{tabular}{|c|c|c|c|c|c|c|}
\hline $\begin{array}{c}\text { Temps } \\
\text { après } \\
\text { infestation }\end{array}$ & 0 & $\mathbf{S}$ & 0 & $\mathrm{~S}$ & 0 & $\mathbf{S}$ \\
\hline 0 & 26 & 12 & 10 & 14 & 29 & 25 \\
\hline $48 \mathrm{~h}$ & 65 & 52 & 63 & 58 & 20 & $+\cdots$ \\
\hline $\begin{array}{l}65 \mathrm{~h} \\
72 \mathrm{~h}\end{array}$ & $\overline{45}$ & 20 & $\overline{41}$ & $\overline{25}$ & 33 & 20 \\
\hline $89 \mathrm{~h}$ & - & - & $\underline{-1}$ & - & 33 & 14 \\
\hline $6 \mathrm{j}$ & - & - & 18 & 14 & 15 & 17 \\
\hline 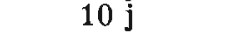 & 16 & 11 & 一 & - & 一 & $\cdots$ \\
\hline $\begin{array}{c}\text { Début } \\
\text { du traitement }\end{array}$ & \multicolumn{2}{|c|}{$\begin{array}{c}5 \text { jours } \\
\text { avant infestation }\end{array}$} & \multicolumn{2}{|c|}{$\begin{array}{l}4 \text { jours } \\
\text { avant infestation }\end{array}$} & \multicolumn{2}{|c|}{$\begin{array}{l}4 \text { jours } \\
\text { avant infestation }\end{array}$} \\
\hline $\begin{array}{c}\text { Infestation } \\
\text { (larves) }\end{array}$ & \multicolumn{2}{|c|}{$12.10^{3}$} & \multicolumn{2}{|c|}{$12.10^{3}$} & \multicolumn{2}{|c|}{$12.10^{3}$} \\
\hline
\end{tabular}

\section{Discussion}

Le traitement par le sulpiride est susceptible d'influencer le parasitisme et l'hypercorticostéronémie réactionnelle.

Les résultats dépendent de la posologie et leur interprétation impose une comparaison avec ceux obtenus lors d'une lactation prolongée.

1 - Importance de la posologie. A priori, il peut paraître surprenant qu'en passant de 10 à 25 , puis à $50 \mathrm{mg} / \mathrm{kg}$, le retentissement sur le parasitisme décroisse progressivement et s'annule pour la dose la plus élevée.

Ce phénomène peut s'expliquer par la pharmacodynamie du sulpiride qui consiste en un blocage des récepteurs à la dopamine. Il en résulte des conséquences multiples dont l'une est l'effet prolactinogène auquel la ratte est particulièrement sensible. La libération de prolactine par l'adénohypophyse est contrôlée par des neurones dopaminergiques; le sulpiride bloquerait les récepteurs à la dopamine, soit au niveau des neurones hypothalamiques responsables de la sécrétion du facteur inhibiteur de la sécrétion de prolactine, déprimant ainsi la libé1ation de ce facteur, soit au niveau des cellules à prolactine de l'antẻhypophyse. En raison de la diversité des récepteurs dopaminergiques, dont le blocage varie avec les doses de sulpiride (Jenner et Marsden, 1979), on peut concevoir que son action suppressive sur les différents systèmes dopaminergiques fasse apparaître pour certaines doses, à côté de l'activité prolactinogène, d'autres actions qui masquent les effets de la prolactine au niveau du parasitisme.

2 - Activités comparées du sulpiride et de la lactation. Nous envisageons deux aspects : le parasitisme et la corticostéronémie réactionnelle au parasitisme. 
- Parasitisme. D'après les travaux de Bailenger et Cabannes (1976), la lactation diminue considérablement l'intensité du parasitisme, retarde le début du déparasitage et ralentit celui-ci. Du retard apporté au commencement du déparasitage, il résulte un maintien de l'élimination des œufs à une valeur proche de son maximum pendant une période s'étalant des environs du $7^{\mathrm{e}}$ au $10^{\mathrm{e}}$ jour. Cette évolution contraste avec celle qui se produit chez les rattes vierges et qui est caractérisée par un pic maximum d'élimination limitée, selon les expériences, au $6 \mathrm{e}$ ou au $7 \mathrm{e}$ jour.

Avec le traitement par le sulpiride $(10 \mathrm{mg} / \mathrm{kg})$, on retrouve l'inhibition du parasitisme et le retard du début du déparasitage se traduisant par un plateau d'élimination maximale des œufs, des environs du $7^{\mathrm{e}}$ aux environs du $10^{\mathrm{e}}$ jour. Par contre, le déparasitage s'achève en même temps que chez les animaux non traités.

Le traitement par le sulpiride ne reproduit donc que partiellement les conséquences de la lactation. Deux explications peuvent être envisagées. En premier lieu, la lactation ne retentit sur le parasitisme que si le nombre de petits est suffisant (Connan, 1970), ce qui suppose qu'un seuil de prolactine est indispensable ; or, il est difficile de reproduire artificiellement une sécrétion de prolactine semblable à celle qui est obtenue pendant l'allaitement et les répercussions atténuées du traitement par le sulpiride peuvent ainsi s'expliquer. En second lieu, il est à noter que le traitement influence seulement le début du parasitisme (intensité maximale, retard du début du déparasitage), mais n'agit pas en fin de déparasitage ; ceci peut s'expliquer en sachant que l'action du sulpiride sur les récepteurs dopaminergiques subit des changements qualitatifs et quantitatifs lorsque le traitement est prolongé (Jenner et coll., 1982).

- Corlicostéronémie. L'effet tampon de la lactation sur la sécrétion des glucocorticoïdes, mise en évidence par Thoman et coll. $(1968,1970)$, inhibe la réaction d'hypercorticostéronémie qui se produit normalement au début de l'évôlution de Strongyloides ralti. Cette répercussion de la lactation peut être attribuée à la prolactine, car il est démontré que cette hormone diminue la réponse corticosurrénale à une agression (Schlein, 1974).

Les injections de sulpiride n'empêchent pas l'hypercorticostéronémie réactionnelle au parasitisme mais atténuent son intensité et en limitent la durée. De même, Rollet (1976) note que ce médicament entraîne une diminution de la réactivité surrénale à un stress.

La lactation et le traitement par le sulpiride, d'action prolactinique, ont donc deux conséquences communes sur le parasitisme : diminution du nombre des adultes qui s'établissent dans l'organisme et retard du début du déparasitage.

La diminution du nombre des larves, qui après leur migration tissulaire s'installent dans la muqueuse intestinale et parviennent à maturité, peut s'expliquer par une meilleure réaction inflammatoire qui s'oppose au développement des larves. Elle résulte d'une inhibition de l'hypercorticostéronémie réactionnelle au stress de l'infestation. Cette altération de la fonction corticosurrénale peut être 
attribuée à la prolactine qui diminue la réponse corticosurrénale à une agression (Schlein, 1974).

Le retard du début du déparasitage relève d'une immuno-dépression caractéristique de la lactation et que le traitement au sulpiride reproduit. Ces deux états ont un dénominateur commun qui est la prolactine. Ces résultats confirment ceux de Connan (1973), qui retardent le déparasitage des ovins infestés par Trichostrongylus en augmentant leur prolactinémie avec des injections de maléate d'acépromazine, ainsi que ceux de Kelly et Dineen (1973) qui obtiennent les mêmes effets en injectant de la prolactine ovine à des rats mâles castrés parasités par Nippostrongylus brasiliensis. Pour ces derniers auteurs, la prolactine agirait en affectant le composant lymphoïde immuno-spécifique du rejet des vers.

\section{Conclusion}

EN CONCLusion, les similitudes relevées chez les rattes parasitées par Strongyloides ratti, au cours de la lactation et du traitement par le sulpiride (molécule prolactinogène) apportent un argument en faveur de l'hypothèse attribuant à la prolactine un rôle fondamental dans les modifications du parasitisme induite par l'état physiologique de l'allaitement, sans préciser par quel mécanisme intervient cette hormone.

\section{BIBLIOGRAPHIE}

Bailenger J., Cabannes A. : Action de la lactation sur le parasitisme des rats par Strongyloides ratti. Ann. Parasitol. Hum. Comp., 1976, 51, 563-576.

Bailenger J., GuY M. : Interactions de deux parasitoses associées chez le Rat : Plasmodium berghei et Strongyloides ratti. Ann. Parasitol. Hum. Comp., 1982, 57, 513-526.

Birkeland S. A., Kristoffersen K. : Lymphocyte transformation with mitogens and antigens during normal human pregnancy: a longitudinal study. Scand. J. Immunol., 1980, 11, 321-325.

Bray R. S., Anderson M. J. : Falciparum malaria and pregnancy. Trans. Roy. Soc. Trop. Med. Hyg., 1979, 73, 427-431.

Bullmer R., Hancock K. W. : Depletion of circulating T lymphocytes in pregnancy. Clin. Exp. Immunol., 1977, 28, 302-305.

Burrells C., Wells P. W., Sutherland A. D. : Reactivity of ovine lymphocytes to phytohaemagglutinin and pokewees mitogen during pregnancy and in the immediate post-parturient period. Clin. Exp. Immunol., 1978, 33, 410-415.

Chaouat G., Voisin C. A. : Regulatory T cell sub-populations in pregnancy. I. Evidence for suppressive activity of the early phase of the MLR. J. Immunol., 1979, 122, 1383-1388.

Chaouat G., Voisin G. A. : Regulatory T cells in pregnancy. V. Allopregnancy-induced T-cellsuppressor-factor is $\mathrm{H}-2$ restricted and bears Ia determinants. Cell. Immunol., 1981, 62, 186-195.

Chaouat G., Voisin G. A., Escalier D., Robert P. : Facilitation reaction (enhancing antibodies and suppressor cells) and rejection reaction (sensitized cells) from the mother to the paternal antigens of the conceptus. Clin. Exp. Immunol., 1979, 35, 13-24.

Clemens L. E., Silteri P. K., Stites D. P. : Mechanism of immunosuppression of progesterone on maternal lymphocyte activation during pregnancy. $J$. Immunol., 1979, 122, 1978-1985.

Clemens J., Smalstig E., Sawyer B. : Antipsychotic drugs stimulate prolactin secretion prolactin release. Psychopharmacologia, 1974, 40, 123-127.

Connax R. M. : The effect of host lactation on the "self-cure " of Nippostrongylus brasiliensis in rats. Parasitology, $1970,61,27-33$.

Gonnan R. M. : The effect of host lactation on a second infection of Nippostrongylus brasiliensis in rats. Parasitology, 1972, 64, 229-233.

Connan R. M. : The immune response of the lactating Rat to Nippostrongylus brasiliensis. Immunology, 1973, 25, 261-267. 
Connan R. M. : Effect of lactation on the immune response to gastrointestinal nematodes. Vet. Rec., 1976, 99, 476-477.

Crofton H. D. : Nematodes parasite populations in sheep on lowland farms. V. Further observations on the post parturient rise and a discussion of its signiflcance. Parasitology, 1958, $48,243$.

DiNeEN J. K., KELLY J. D. : The suppression of rejection of Nippostrongylus brasiliensis in lactating rats: the nature of the immunological defect. Immunology, 1972, 22, 1-12.

Iwasaki Y., Kato Y., Chiara K., OHgo K., MaEda K., ImURa H. : Effect of sulpiride on plasma prolactin in rats. Neuroendocrinology, 1976, 21, 267-274.

Jenner P., Hall M. D., Murugaiah K., Rupniak N., Theodorou A., Marsden C. D. : Repeated administration of sulpiride for three weeks produces behavioural and biochemical evidence for cerebral Dopamine receptor supersensitivity. Biochem. Pharmacol., 1982, 31, 325-328.

Jenner P., Marsden C. D. : Mécanisme d'action des benzamides substituées. Psychologie Med., $1979,11,2775-2793$.

KElly J. D., Dineen J. K. : The suppression of rejection of Nippostrongylus brasiliensis in Lewis strain rats. Immunology, 1973, 24, 551-558.

I.anza M., PiCard D., Carlon N. : Effets du sulpiride sur le tractus génital, la glande mammaire et l'hypophyse antérieure de la ratte. C. R. Soc. Biol., 1971, 169, 1365-1368.

McLEOD R., RoByn Cl. : Mechanism on increased prolactin secretion by sulpiride. J. Endocrinol., 1977, 72, 273-277.

Mancini A. M., Guitelman A., Vargas C. A., Debeljuk L., Aparicio N. J. : Effect of sulpiride on serum prolactin levels in humans. $J$. Clin. endocr., 1976, 42, 181-184.

Marchandise B. : Action galactologue du sulpiride. Ann. Endocrinol., 1971, 32, 716-719.

Mori S., FuJI T., Ishida A., Kawakita Y., Kaneda Y., Nagasowa H. : Observations on lactation with sulpiride in female rats. Acta Histochem. Cytochem., 1975, 8, 203-213.

O'Sullivan B. M. : Effects of lactation on Trichostrongylus colubriformis infections in the guinea pig. Int. J. Parasitol., 1974, 4, 177-181.

O'Sullivan B. M., Donald A. D. : A field study of nematode parasite populations in the lactating ewe. Parasitology, 1970, 61, 301-315.

Reuse J. : Pharmacologie du métoclopramide, du sulpiride et de leurs dérivés. Actualités pharmacologiques (Hazard et coll.). Masson, éd., 1979, 99-118.

Rollet J., Czyba J. C., Corrinet D.: Retentissement endocrinien du Sulpiride chez le Hamster doré. Prog. Med., 1976, 104, 89-96.

Ruitenberg E. J., Buys J. : Thymic atrophy during early pregnancy and its effect on a Trichinella spiralis infection in mice, including intestinal pathology and blood eosinophilia. Vet. Immunol. Immunopath., 1980, 1, 199-214.

Schlein P. A., Zarrow M. X., Denenberg V. H. : Le rôle de la prolactine dans l'inhibition de la réponse corticostéroïdienne surrénale chez le Rat. $J$. Endocrinol., 1974, 62, 93-99.

SElBY G. R., WAKELIN D. : Suppression of the immune response to Trichuris muris in lactating mice. Parasitology, $1975,71,77-85$.

Soulairac A., Soulairac M. L., Franchimont P. : Action neuro-endocrinienne du sulpiride et du sultopride sur la fonction sexuelle du Rat. Thérapie, 1975, 30, 597-612.

Stahn R., Fabricius H., Hartleitner W. : Suppression of human T-cell colony formation during pregnancy. Nature, 1977, 276, 831-832.

Stefar Y., Benakis A. : Action du sulpiride sur les cellules à prolactine, la glande mammaire et le tractus génital de rats mâles et femelles, entiers et hypophysectomisés. J. Pharmacol., $1976,7,379-392$.

Szekeres J., Csernus V., PeJtsik S., Emody L., Pacsa A. S. : Progesterone as an immunologic blocking factor in human pregnancy serum. J. Reprod. Immunol., 1981, 3, 333-339.

Suzuki K., Tomasi T. B. : Immune responses during pregnancy. Evidence of suppressor cells for splenic antibody response. J. Exp. Med., 1979, 150, 898-908.

Thoman E. B., Conner R. L., Levine S. : Lactation suppresses adrenal corticosteroid activity and aggressiveness in rats. J. Comp. Physiol. Psychol., 1970, 70, 364-369.

Thoman E. B., Wetzel A., Levine S. : Lactation prevents disruption of temperature regulation and suppresses adreno-cortical activity in rats. Commun. Behav. Biol., 1975, 2, 165-171.

Truycotte J. G., Haine R. F., Brody G. L., Mayen T. J., Schwartz S. A. : Immunosuppression by medroxyprogesterone acetate. Transplantation, 1968, 6, 248-257.

Van Zon A. A. J. C., Eling W. M. C. : Depressed malarial immunity in pregnant mice. Infect. Immun., 1980, 28, 630-632. 